1997-12-01

\title{
Unfolding of the period-two bifurcation in a fiber laser pumped with two modulation tones
}

\author{
Scott Glasgow \\ T. C. Newell \\ A. Gavrielides \\ V. Kovanis \\ D. Sukow
}

Glasgow@mathematics.byu.edu

See next page for additional authors

Follow this and additional works at: https://scholarsarchive.byu.edu/facpub

Part of the Astrophysics and Astronomy Commons, and the Physics Commons

\section{Original Publication Citation}

Physical Review E, vol 56, no 6, pp 7223-7231.

\section{BYU ScholarsArchive Citation}

Glasgow, Scott; Newell, T. C.; Gavrielides, A.; Kovanis, V.; Sukow, D.; and Erneux, T., "Unfolding of the period-two bifurcation in a fiber laser pumped with two modulation tones" (1997). Faculty Publications. 656.

https://scholarsarchive.byu.edu/facpub/656

This Peer-Reviewed Article is brought to you for free and open access by BYU ScholarsArchive. It has been accepted for inclusion in Faculty Publications by an authorized administrator of BYU ScholarsArchive. For more information, please contact ellen_amatangelo@byu.edu. 


\section{Authors}

Scott Glasgow, T. C. Newell, A. Gavrielides, V. Kovanis, D. Sukow, and T. Erneux 


\title{
Unfolding of the period-two bifurcation in a fiber laser pumped with two modulation tones
}

\author{
T. C. Newell, A. Gavrielides, V. Kovanis, ${ }^{*}$ and D. Sukow ${ }^{\dagger}$ \\ Nonlinear Optics Group, Phillips Laboratory, Kirtland Air Force Base, New Mexico 87117-5776 \\ T. Erneux and S. A. Glasgow \\ Université Libre de Bruxelles, Optique Nonlinéaire Théorique, Campus Plaine, Case Postale 231, 1050 Bruxelles, Belgium
}

(Received 20 January 1997)

\begin{abstract}
The effect of a small second frequency component on a pump modulated neodymium fiber laser is investigated experimentally and theoretically. This term, whose frequency is exactly half the primary driver, incites an unfolding of the attractor. It breaks the period two pitchfork bifurcation and splits the period one orbit. The modification of the bifurcation diagram is studied analytically by employing a map derived from the class $B$ laser rate equations. We determine specific conditions and scaling laws for this phenomenon. Our analytical predictions are in good agreement with recorded experimental data. [S1063-651X(97)02307-6]
\end{abstract}

PACS number(s): 42.65.Sf, 42.55.Wd, 05.45.+b

\section{INTRODUCTION}

Class $B$ lasers [1] that are modulated either through the pump or through intracavity losses have often been used for investigations of nonlinear dynamics in optical systems. One such system, an electro-optic modulated $\mathrm{CO}_{2}$ laser, was originally used to experimentally observe chaos [2]. Among the impressive array of other research, acousto-optic loss modulated $\mathrm{CO}_{2}$ lasers have also been used to study multistability [3] and measure Floquet multipliers by targeting unstable periodic orbits [4]. Potential applications of this research include the stabilization of unstable steady states of lasers [5] and the use of chaotic signals for communication applications [6,7].

A topic of exceptional relevance is the behavior of nonlinear systems at the period two (P2) and higher period bifurcation points. Typically, the bifurcation parameter is the amplitude of the $2 \pi / \omega$ periodic modulation term. As the system approaches the bifurcation point, stochastic and deterministic influences can be strongly amplified, as suggested by Wiesenfeld and McNamara [8]. This idea was subsequently tested in a NMR laser [9]. Recent investigations incorporated loss modulated $\mathrm{CO}_{2}$ lasers with a driving frequency $\omega$, and a signal frequency at $\omega / 2$ [10-12]. These investigations yielded insights in the (de)amplification of the $\omega / 2$ frequency component of the Fourier spectra. Additionally, it is found that a $-2 / 3$ scaling law exists between the gain of the $\omega / 2$ frequency component and the amplitude of the signal frequency just below the P2 bifurcation point. The use of an $\omega / 2$ component to control chaos has also been investigated theoretically and experimentally [13-17].

This natural relation of nonlinear systems with their resonance frequencies remains a source of insightful studies. In this paper we continue the investigation of periodic perturbations. We consider a $2 \pi / \omega$ periodic pump modulated fiber

\footnotetext{
*Also at Department of Mathematics and Statistics, University of New Mexico, Albuquerque, NM 87131.

${ }^{\dagger}$ Also at Department of Physics, Duke University, P.O. Box 90305, Durham, NC 27708.
}

laser and investigate the effect a second small $\pi / \omega$ periodic modulation component has at the P2 bifurcation point. This term has a strong amplification effect on the P2 pitchfork bifurcation [18] and allows control of the P2 instability [19]. We present experimental data obtained from time domain measurements that shows how the P2 bifurcation point is affected. We also show that a map derived from the class $B$ laser rate equations accurately predicts such behavior. Though this map was initially derived for a loss modulated class $B$ laser [20], we find that the pump modulated case behaves similarly. Consequently, we can expect that the pump modulated class $B$ laser should be similar to the loss modulated case. Our results are in agreement with the previous studies of two frequency loss modulated $\mathrm{CO}_{2}$ lasers $[10,11]$.

The response of the driven fiber laser can be studied numerically by a two polarization model $[21,22]$ and its relevance for two tone modulation experiments is currently being investigated. Particular attention is devoted to the global changes of the bifurcation diagram (shifts of bifurcation points, periodic windows, crises) resulting from the second modulation. These are specific to the fiber laser and depend on the laser parameters [23]. In contrast, we concentrate on the changes of the bifurcation diagram near the first P2 bifurcation point. Our objective is to determine analytically and verify experimentally all bifurcation effects that are introduced by the additional $\omega / 2$ modulation. Specifically, our analysis leads to simple scaling laws describing the changes of the bifurcation points and the effect of the relative phase between the $\omega$ and the $\omega / 2$ modulations. Because our theory is a local theory, our results are relevant for a large class of periodically modulated lasers as well as other periodically forced nonlinear oscillators.

In order to analyze the bifurcation problem both qualitatively and quantitatively, we consider the equations of a class $B$ laser. We then derive equations for a map that allow a simple analytical description of the P2 bifurcation and the effect of the $\omega / 2$ component. These results compare remarkably well with our experimental observations and clarify previous observations based on $\mathrm{CO}_{2}$ laser experiments.

This paper is organized as follows. In Sec. II, the experi- 


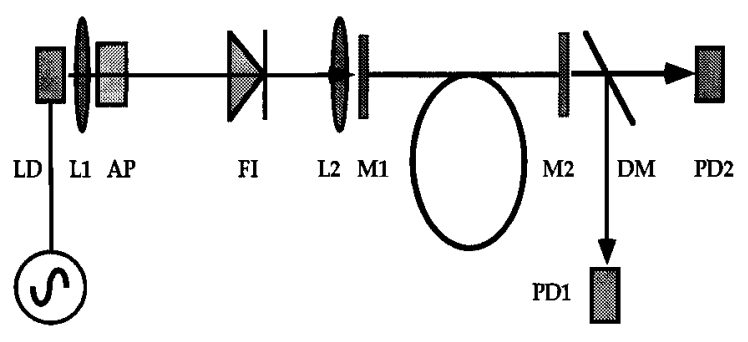

FIG. 1. The experimental arrangement. LD, laser diode; LI, collimating lense; AP, anamorphic prism; F1, Faraday isolator; L2, focusing lense; M1, input coupling mirror; M2, 95\% reflective output coupling mirror; DM, dichroic mirror (HR at $810 \mathrm{~nm}$, HT at $1064 \mathrm{~nm})$; PD1, PD2 photodetectors.

mental arrangement is described. In Sec. III, observations of the two tone modulated fiber laser are presented. Constructed from recorded data, bifurcation diagrams show the drastic consequences of the small second tone. In Sec. IV a model derived from the rate equations is introduced. Computational and analytical fixed point studies are then described in detail. A summary and discussion of the problem is found in Sec. $\mathrm{V}$. Details of the derivation of the map are located in Appendixes $\mathrm{A}$ and $\mathrm{B}$.

\section{EXPERIMENTAL SETUP}

A schematic of the neodymium fiber laser is shown in Fig. 1. The primary component is a $2.8 \mathrm{~m}$ single transverse mode doped optical fiber whose cleaved ends are butted to input and output coupling mirrors. The input dichroic mirror is highly transmissive at the pump wavelength of $810 \mathrm{~nm}$ and highly reflective at the lasing wavelength of $1088 \mathrm{~nm}$. The output coupler has a reflectivity of $95 \%$ at $1088 \mathrm{~nm}$. The fiber is $3.5 \mu \mathrm{m}$ in diameter and has an absorption rate of 8.5 $\mathrm{db} / \mathrm{m}$ at $810 \mathrm{~nm}$. The cavity decay time and the fluorescence lifetime are $\tau_{c}=11.1 \mathrm{~ns}$ and $\tau=460 \mu \mathrm{s}$, respectively. In addition to the large number of longitudinal modes oscillating, there are two polarization states. The laser is pumped with an SDL-5412 laser diode operating at $810 \mathrm{~nm}$. The initially elliptical beam is collimated, rounded with an anamorphic prism pair then reduced by means of a simple telescope in order to mode match with the focusing lens and fiber. Minute reflections from the cleaved surface of the fiber that are coupled back into the modulated laser diode render its intensity highly erratic. These detrimental reflections were sufficiently negated by incorporating a Faraday isolator tuned to obtain over $43 \mathrm{~dB}$ attenuation. On the output end of the laser, the unabsorbed pump beam is separated from the fiber light by a dichroic mirror then steered into a photodetector. The laser light propagates through a long wave pass filter $(950$ nm cutoff) into a New Focus $1811125 \mathrm{MHz}$ bandwidth photodetector. Intensity time series are recorded with a Tektronix RTD710 ten-bit vertical resolution digitizer. The pump beam is modulated at a frequency, $f=30 \mathrm{kHz}$. At this pump level the relaxation oscillations of the free running laser were measured at $f_{r}=40 \mathrm{kHz}$. We modulated the current of the laser diode with

$$
I(t)=I_{0}+I_{1} \cos (\omega t)+I_{2} \cos \left(\frac{1}{2} \omega t+\phi\right) .
$$
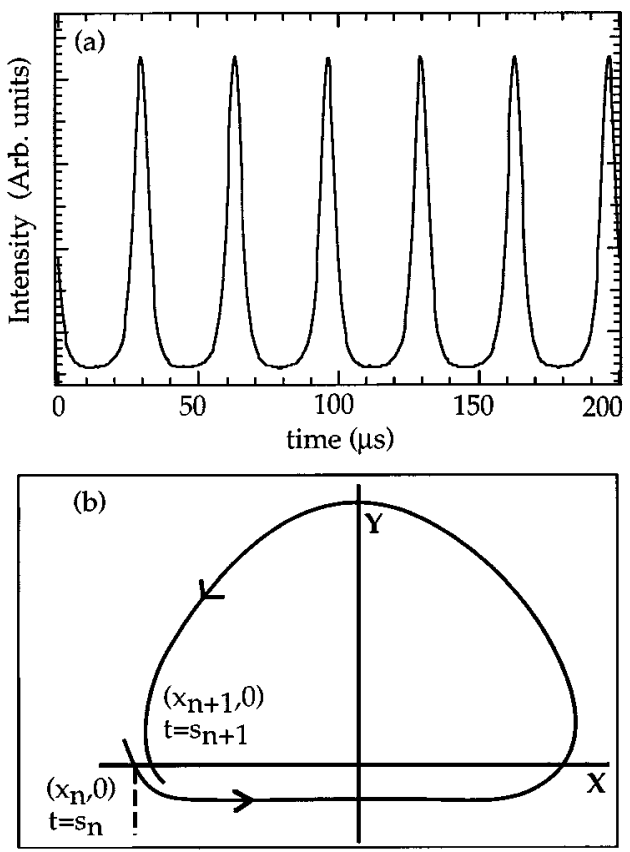

FIG. 2. (a) An intensity time series of the single frequency pump modulated neodymium fiber laser. (b) A phase portrait from which the map is derived. The $X(Y)$ axis represents the dimensionless inversion (intensity). Point $(x, y)=\left(x_{n}, 0\right)$ at time $t=s_{n}$, denotes the end of a pulse and begining of a period in which the population inversion increases while the intensity is negligible. After the subsequent intensity spike, the laser relaxes into a state $\left(x_{n+1}, 0\right)$ at time $t=s_{n+1}$.

The threshold of the fiber laser occurs at $I_{\text {th }}=34 \mathrm{~mA}$ pump current, and we operate the laser with $I_{0}=65 \mathrm{~mA}$. This gives a pump parameter of $A_{0} \equiv I_{0} / I_{\mathrm{th}}=1.93$.

$I_{1}, I_{2}$, and $\phi$ constitute the control parameter space we explore below. Once the pump is even slightly modulated $\left(I_{1} \ll I_{0}\right)$, the normal $\mathrm{cw}$ output of the fiber laser is replaced by large amplitude relatively spiky oscillations. A typical time series of the intensity is shown in Fig. 2(a). The laser is quiet for relatively long amounts of time followed by strong pulses.

There is a degree of uncertainty in the exact amount of laser light coupled into the fiber. We found that the laser diode intensity, when modulated at $40 \mathrm{kHz}$, is linearly related to its driving current. Thus we leave the modulation measurements in terms of the laser diode current. We measure the total intensity of the fiber laser and leave the obtained time series in the ten-bit integer scale of the digitizer.

\section{EXPERIMENTAL OBSERVATIONS}

In this section we study the dynamics of the fiber laser in a primary modulation amplitude $I_{1}$, second frequency amplitude $I_{2}$, and phase difference $\phi$ control parameter space. In particular, we focus on the response of the laser when it is operating in the vicinity of its $\mathrm{P} 2$ bifurcation point.

For an initial experiment, we fix $I_{2}$ and $\phi$ and study the dynamics as $I_{1}$ is ramped from $2.1 \mathrm{~mA}$ to $2.5 \mathrm{~mA}$. At each increment, intensity time series are digitized. From the recorded data, the maxima of the intensity oscillations are extracted and plotted versus the primary modulation amplitude 


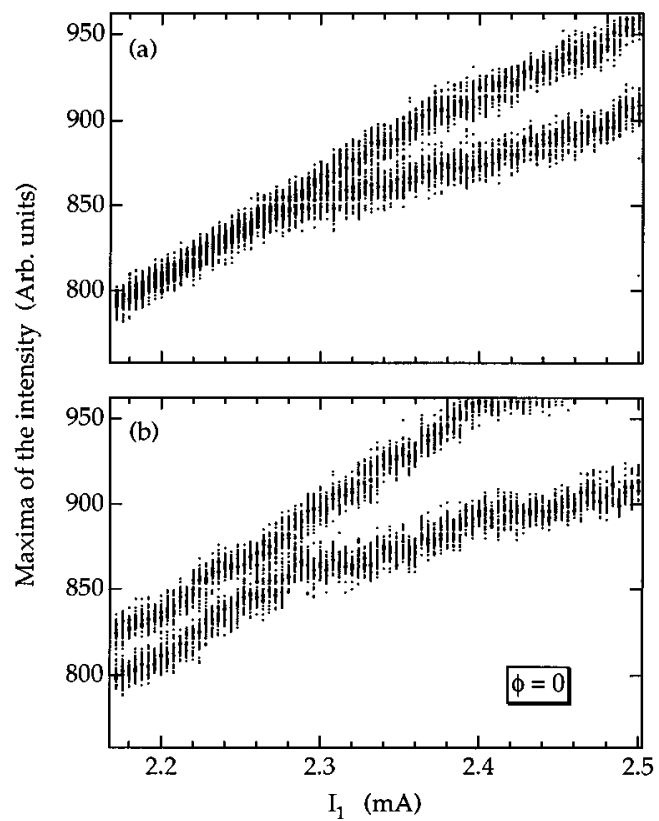

FIG. 3. (a) Experimental bifurcation diagram of the fiber laser with one tone pump modulation. (b) Two modulation driving. $I_{2}=0.06 \mathrm{~mA}$ and $\phi=0$. Though $I_{2}$ is very small, the P1 orbit is completely split.

$I_{1}$. The resulting bifurcation diagrams are shown in Figs. 3 and 4. In Figs. 3(a) and 4(a) $I_{2}=0 \mathrm{~mA}$. These two figures are single modulation frequency bifurcation diagrams to be used as a reference. The focus is upon the $\mathrm{P} 2$ pitchfork bifurcation that occurs at $I_{1} \approx 2.4 \mathrm{~mA}$. Note that there is a slight difference in the bifurcation point between the two figures. This is predominantly due to environmental factors that affect the system. Consequently, in the experiment, care was taken to first record a reference data set then immediately acquire the

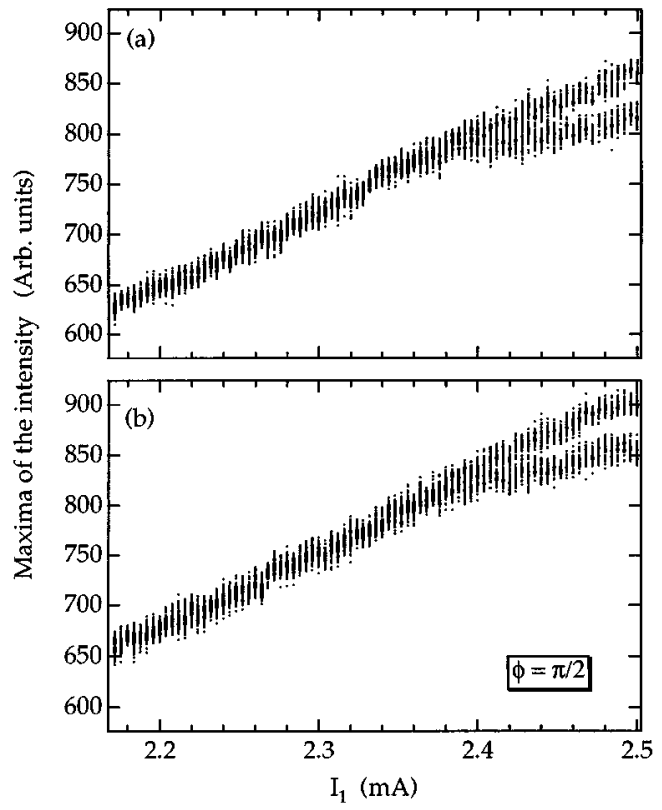

FIG. 4. (a) One tone modulation reference bifurcation diagram. (b) $I_{2}=0.06 \mathrm{~mA}$ and $\phi=\pi / 2$. At this critical phase the effect due to the perturbation is negligible.

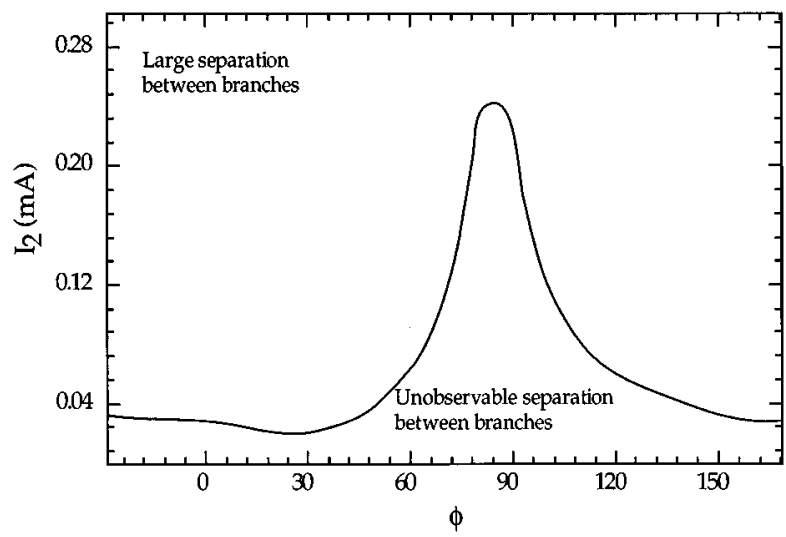

FIG. 5. This contour plot, constructed in a $I_{2}$ and $\phi$ control space, shows the effect of the second tone on the dynamics of the system. Regardless of $I_{2}$, at $\phi=\pi / 2$, the effect due to the perturbation is minimized.

set in which the perturbation was applied. The environmental factors, ambient temperature and mechanical drift, affect the fiber laser on a very slow time scale, tens of minutes versus microseconds that the observed dynamics take place. Therefore, these factors do not influence the fundamental bifurcation mechanisms. Figure 3(b) shows the bifurcation diagram for $I_{2}=0.06 \mathrm{~mA}$ and $\phi=0$. Here no true P1 orbit exists since $I_{2}$, albeit small, is still sufficient to unfold the P2 pitchfork bifurcation. Consequently, the formerly P1 orbit splits. We find that at $\phi=0$, the splitting of the P1 orbit due to the second frequency component is maximized. In contrast, Fig. $4(\mathrm{~b})$ is a bifurcation diagram taken for $\phi=\pi / 2$ and $I_{2}=0.006 \mathrm{~mA}$. On comparison with Fig. 4(a), little change in the bifurcation point or the P1 orbit occurs.

Examining the drive signal Eq. (2.1) provides clues to the relationship between the effect due to the second tone and $\phi$. At a phase angle $\phi=0$ the amplitudes of successive maxima will be different by $2 I_{2}$. In contrast, at $\phi=\pi / 2$ the amplitudes of successive maxima are equal. Consequently, we expect that the response of the laser will be drastically changed if $\phi=0$.

In the next experiment we probe the $I_{2}-\phi$ control space in order to study the lasers response to variations in the $\omega / 2$ drive component. The goal is to distinguish the values of $I_{2}$ and $\phi$ that have a clear effect on the system from those values that, at least experimentally, have little effect. Here $I_{1}=2.35 \mathrm{~mA}$, that is, just below the P2 point when $I_{2}=0$. The procedure consists of a computer controlled doubly iterative loop in $\phi$ and $I_{2}$. The inner loop ramps $\phi$ from $-25^{\circ}-165^{\circ}$ while the outer loop increments $I_{2}$. At each point, a time series is acquired and the maxima of each oscillation extracted. We then calculate the average deviation between the $N$ th and $N+j$ th peak where " $j$ " refers to the period of the orbit in question and $N$ is summed over all peaks. This experiment is summarized in the contour plot shown in Fig. 5. In this figure we distinguish the region where the effect of the perturbation is small and obscured by the inherent system noise (so that the trajectory resembles a $\mathrm{P} 1$ orbit) from the region where a split P1 orbit is experimentally discernable. The contour line marks the boundary where we are able to distinguish the split of the P1 orbit from the collected data. This plot is intended to provide insight into 
the effect of the perturbation as a function of $\phi$. For small values of $I_{2}$, the effect of the perturbation is obscured, and the laser operates in what resembles a P1 orbit. As $I_{2}$ is increased, the splitting of the P1 orbit becomes apparent and the laser operates in a $\mathrm{P} 2$ regime. However, in the vicinity of $\phi=\pi / 2$, the effect of the perturbation is strongly attenuated regardless of $I_{2}$.

In a final experiment, we investigate the amplification of the $\omega / 2$ frequency component nearby but below the P2 bifurcation point. The gain $G$ of this natural amplifier is defined to be the ratio of the $\omega / 2$ frequency component when $I_{1} \approx I_{p d}$ to this component when $I_{1}=0[10,11]$

$$
G=\frac{F\left(\omega / 2, I_{1} \approx I_{p d}\right)}{F\left(\omega / / 2, I_{1}=0\right)}
$$

If $I_{2}$ is small, the scaling of the denominator goes as $I_{2}$. When $10^{-3}<I_{2} / I_{p d}<5 \times 10^{-2}$ and $\phi=0$, we find that the numerator scales as $I_{2}^{1 / 3}$. Consequently, the gain as a function of $I_{2}$ scales as $I_{2}^{-2 / 3}$ in this region. Thus there is a sizable gain of this $\omega / 2$ component that decreases as $I_{2}$ increases. However, for larger values of $I_{2}$ or if the system is not close to the $\mathrm{P} 2$ bifurcation point, we find that the numerator responds linearly. The laser then functions as a noninverting unity gain amplifier of the $\omega / 2$ frequency component.

\section{INVESTIGATING THE CLASS $B$ LASER MAP}

In this section, we analyze the bifurcation diagram of the periodic states in the vicinity of the P2 bifurcation point. Though the fiber laser is a complex system that cannot be completely described as a class $B$ laser, the bifurcation changes near the $\mathrm{P} 2$ bifurcation point and resulting from the $\omega / 2$ component do not depend on the details of the model but rather the nature of the bifurcation transition (here a supercritical P2 pitchfork bifurcation). In order to determine these bifurcation effects, we shall concentrate on the well known single mode laser rate equations and derive simple equations for a map. The derivation of the map is motivated by our numerical and experimental observations of strongly pulsating intensity oscillations consisting of short and intense pulses separated by relatively large periods of time in which the laser intensity is negligible, see Fig. 2(a). The model equations are the single mode class $B$ laser equations for the amplitude of the laser field $E$ and the inversion of population $N$

$$
\begin{gathered}
\frac{d E}{d t}=(N-1) E, \\
\frac{d N}{d t}=\gamma\left[A(t)-N-N|E|^{2}\right] .
\end{gathered}
$$

The time $t$ is measured in units of the cavity decay time, $\tau_{c}$ and $\gamma$ is the ratio of the cavity decay time to the fluorescence lifetime, $\tau\left(\gamma \equiv \tau_{c} / \tau \simeq 2.4 \times 10^{-5}\right)$. $A(t)$ is the pump parameter and is given by

$$
A(t)=A_{0}+A_{1} \cos \left(\omega_{1} t\right)+A_{2} \cos \left(\omega_{2} t+\phi\right),
$$

where $A_{0}>1$ and $\omega_{2}=\omega_{1} / 2$. In Eq. (4.3), the modulation frequency $\omega_{1}$ is close to the free running laser relaxation frequency defined by $\omega_{R} \equiv \sqrt{2 \gamma\left(A_{0}-1\right)}$ (hence the lasing threshold occurs for $A_{0}=1$ ). The amplitudes $A_{1}$ and $A_{2}$ are positive $\left(A_{2} \ll A_{1} \ll A_{0}\right)$ and $\phi$ is a phase. We consider $A_{1}$ as our bifurcation parameter and keep $A_{2}$ and $\phi$ fixed. Of particular interest is the modification of the laser response near the P2 bifurcation point as $\phi$ is progressively changed.

The equations for this map are obtained by using the method developed for a loss modulated class $B$ laser by Schwartz and Erneux [20]. Appendix A outlines the derivation of the pump modulated map along with definitions of the notation used below. As a visual aid for the construction of the map, refer to Fig. 2(b). Consider a space where the $X$ and $Y$ axes represents the dimensionless inversion and intensity, respectively. Defining $x$ as the scaled inversion and $y$ as the scaled intensity, we start from the point $(x, y)=\left(x_{n}, 0\right)$ at time $t=s_{n}$ which describes the state of the laser as a pulse in ending. The laser is quiescent up to $t=s_{n+1}$ at which point the subsequent pulse occurs. Because the intensity is constant in this region, we analytically solve Eq. (4.2). After the ensuing pulse, considered short enough in time so that the drive terms are essentially constant, the laser relaxes into the state $(x, y)=\left(x_{n+1}, 0\right)$ at time $t=s_{n+1}$. We then analytically solve this problem in the phase space. The two solutions are then matched using the method of matched asymptotic expansions [24]. The resulting map determines the state of the laser from the end of one pulse to the end of the next. It consists of one equation for the period $s_{n+1}-s_{n}$ and one for $x_{n}$ after an oscillation, see Eq. (A7) and Eq. (A8).

From the map, we compute the set of points $s_{n+1}-s_{n}$ as the bifurcation parameter, $\delta_{1}$ and control parameters $\delta_{2}$ and $\phi$ are varied. These generated points are often referred to as the interspike interval [7] and denote the amount of time lapsed between successive pulses. Curiously these intervals are not necessarily simple harmonics of the driving frequency. Instead the map can exhibit a rich structure equivalent to a Poincaré surface constructed at discrete time intervals. This is due to the fact that interspike intervals of spiky data carry the same dynamical state information as amplitude measurements of the intensity. Below we compute bifurcation diagrams in order to probe the dynamics of the system and then we perform a fixed point analysis. Bifurcation diagrams are shown in Fig. 6. Here $\omega_{1}=0.9$ and $\varepsilon=0.01$ $\left(\epsilon \equiv \sqrt{\gamma\left[2\left(A_{0}-1\right)\right]^{-1}}\right)$. Figure 6(a) shows the reference bifurcation diagram when $\delta_{2}=0$. The interspike interval remains constant up to $\delta_{1}=1.0$ at which point the pitchfork P2 bifurcation occurs. In Fig. 6(b) $\delta_{2}=0.01$ and $\phi=\pi / 2$. The addition of the second frequency component destroys the pitchfork bifurcation. The result is the occurrence of an imperfect bifurcation and the replacement of the $\mathrm{P} 2$ bifurcation point with a limit point [25]. The inner upper and lower trace in Fig. 6(b) represents the numerical solution when $\delta_{1}$ is decreased. These branches arc towards the limit point. In Fig. 6(c) $\delta_{2}=0.01$ and $\phi=171^{\circ}$. At this phase, the unfolding of the attractor is substantially greater.

Subsequently we make a detailed study of the effect of $\phi$ on the system dynamics. The P2 orbit is described in terms of the intervals between intensity spikes $s_{n+1}-s_{n}$ and $s_{n+2}-s_{n+1}$ given by 


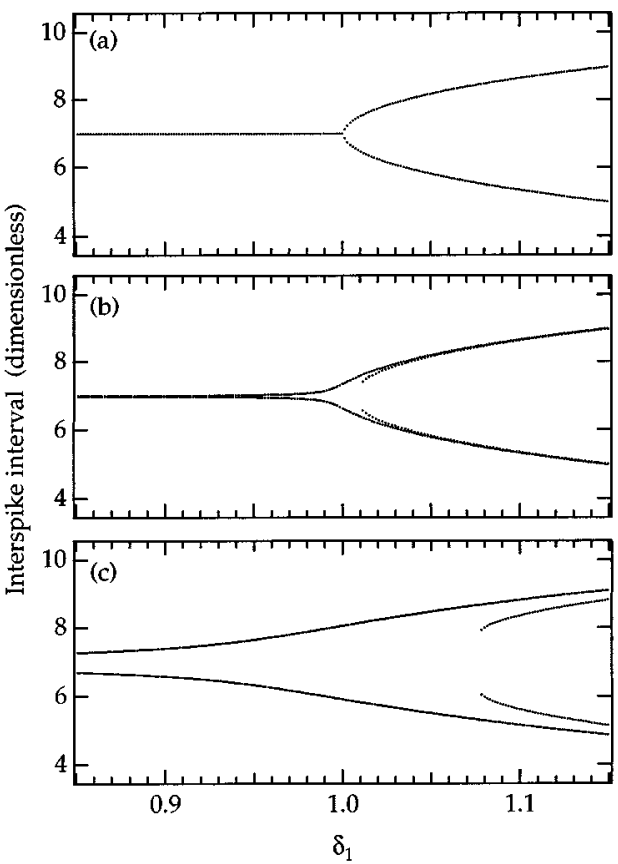

FIG. 6. Numerical bifurcation diagrams of the interspike interval, $s_{n+1}-s_{n}$, vs $\delta_{1}$ (a) $\delta_{2}=0$. A pitchfork bifurcation occurs at $\delta_{1}=1.0$ creating the $\mathrm{P} 2$ orbit. (b) $\delta_{2}=0.01$ and $\phi=90^{\circ}$. The pitchfork bifurcation no longer exists and the P2 bifurcation point is replaced by a limit point located where the two inner branches converge. (c) $\phi=171^{\circ}$ and $\delta_{2}=0.002$. At this phase, the unfolding is substantially larger.

$$
s_{n+1}-s_{n}=\frac{(2 \pi+T)}{\omega_{1}} \text { and } s_{n+2}-s_{n+1}=\frac{(2 \pi-T)}{\omega_{1}} .
$$

Note that for a P1 orbit, $T=0$. In Fig. 7 we plot $T$, versus the phase for various values of $\delta_{2}$ when $\delta_{1}=1.0$. We find that when $\phi=\pi / 2, T$ is minimized. This effect is maximized at

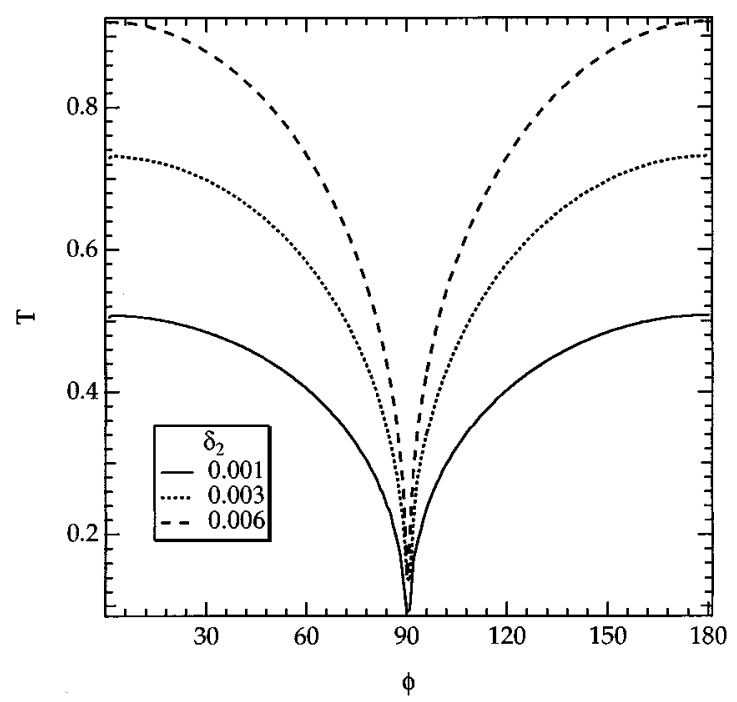

FIG. 7. $T\left[T=\omega_{1}\left(s_{n+1}-s_{n}\right)-2 \pi\right]$ is plotted vs $\phi$ for various values of $\delta_{2}$ when $\delta_{1}=1.0$. The influence of the second tone is maximized when $\phi=0^{\circ}$ or $\phi=180^{\circ}$ and minimized when $\phi=90^{\circ}$. $\phi=0$ and $\phi=\pi$. This figure is in agreement with the experimental findings shown in Fig. 5.

Due to the inclusion of damping, the map described by Eq. (A7) and Eq. (A8) is too complicated to be solved analytically for the P1 orbit and P2 and higher bifurcation points. However, we may benefit from the small value of $\epsilon$. For our fiber laser, with $\gamma \simeq 2.4 \times 10^{-5}$ and $A_{0}=1.93$, Eq. (A4) informs us that $\epsilon \simeq 4 \times 10^{-3}$. The small value of $\epsilon$ suggests that we investigate the equations of the map as $\epsilon=0$. Taking the limit $\epsilon \rightarrow 0$ from Eq. (A7) and (Eq. (A8) leads to the following problem for the period $s_{n+1}-s_{n}$ and the change in amplitude $x_{n+1}-x_{n}$ :

$$
\begin{aligned}
s_{n+1}-s_{n}= & -2 x_{n}+2 \frac{\delta_{1}}{\omega_{1}} \sin \left(\omega_{1} s_{n}\right)+2 \frac{\delta_{2}}{\omega_{2}} \sin \left(\omega_{2} s_{n}+\phi\right), \\
x_{n+1}-x_{n}= & -\frac{\delta_{1}}{\omega_{1}}\left[\sin \left(\omega_{1} s_{n+1}\right)+\sin \left(\omega_{1} s_{n}\right)\right] \\
& -\frac{\delta_{2}}{\omega_{2}}\left[\sin \left(\omega_{2} s_{n+1}+\phi\right)+\sin \left(\omega_{2} s_{n}+\phi\right)\right] .
\end{aligned}
$$

Hidden in the simplicity of Eq. (4.5) is the fact that it accurately mirrors the class $B$ laser rate equations. Equation (4.5) allows us to avoid the computationally intensive task of integrating Eq. (4.2) and to analytically study the various phenomena that arise as a result of the applied perturbations.

We now describe our fixed point analysis. Using Eq. (A1) and Eq. (A9), there is a correspondence between the maximum intensity, $y_{M}$ and $x_{n+1}$ that is given by $y_{M}=\left(A_{0}\right.$ $-1)\left(1+\frac{1}{2} x_{n+1}^{2}\right)$. If $\delta_{2}=0$, the P1 solution satisfies the conditions $s_{n+1}-s_{n}=2 \pi / \omega_{1}$ and $x_{n+1}=x_{n}$. From Eq. (4.5), we then obtain

$$
x_{n}=-\frac{\pi}{\omega_{1}} \quad \text { and } \quad s_{n}=(2 n-1) \frac{\pi}{\omega_{1}} .
$$

From the linear stability analysis of the P1 solution, we find a period doubling bifurcation point at

$$
\delta_{1}=1 .
$$

We wish to investigate the bifurcation diagram of the periodic states in the vicinity of this point and for small values of $\delta_{2}$. We anticipate the effect of the second modulation by assuming that the term multiplying $\delta_{2}$ acts as a imperfection in the bifurcation problem [25]. Its effect is strongest near the bifurcation point and we solve Eqs. (4.5) by a perturbation method valid for small $\delta_{2}$ (see Appendix B). The final result for the P2 orbit is described in terms of $T$ defined in Eq. (4.4). $T=T\left(\delta_{1}\right)$ is determined from the $\mathrm{P} 2$ fixed point equations. If $\delta_{1}-1 \ll \delta_{2}^{2 / 3}, T=O\left(\delta_{2}\right)$ which is small. Similarly if $\delta_{1}-1 \gg \delta_{2}^{2 / 3}, T$ is the combination of the known P2 solution for $\delta_{2}=0$ plus an $O\left(\delta_{2}\right)$ correction [25]. However when $\left|\delta_{1}-1\right|=O\left(\delta_{2}^{2 / 3}\right)$, a significant amplification occurs near the P2 bifurcation point. Specifically, $T=O\left(\delta_{2}^{2 / 3}\right)$ and satisfies to first approximation, Eq. (B14), or equivalently,

$$
2\left(\delta_{1}-1\right) T-\frac{1}{12} T^{3}+8 \delta_{2} \sin (\phi-\pi / 2)=0 .
$$




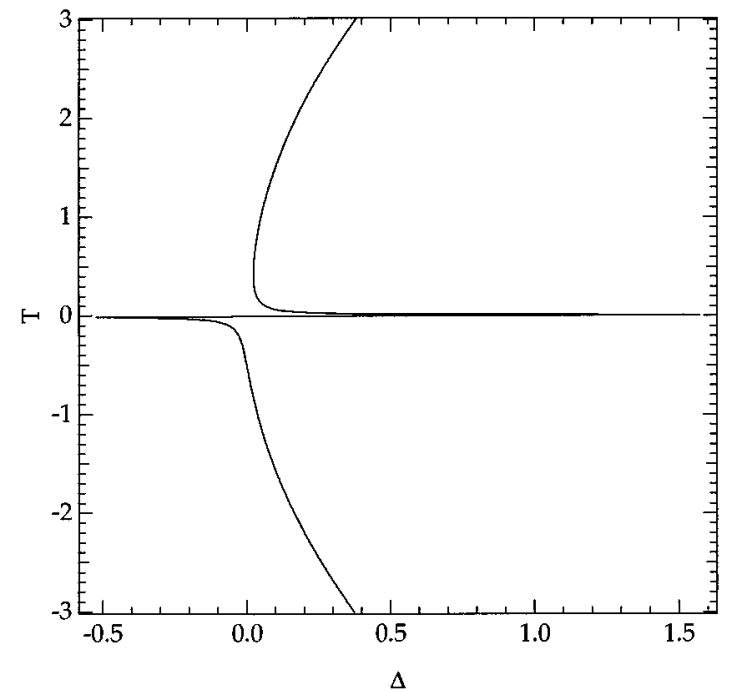

FIG. 8. An analytical calculation of $T$ vs the control parameter $\Delta$. This plot shows that the perturbation unfolds the attractor. The consequence is the destruction of the P2 pitchfork bifurcation. Instead, the second (upper) branch is now bounded by a limit point that is defined in Eq. (4.9).

The solution of this equation is best analyzed in terms of the implicit solution $\delta_{1}=\delta_{1}(T)$. If $\phi \neq \pi / 2$, the P2 pitchfork bifurcation is destroyed and replaced by a smooth transition branch and a second branch bounded by a limit point, see Fig. 8. The limit point can be determined from Eq. (4.8) and is located at

$$
\delta_{1}=1+\frac{1}{2}\left[6 \delta_{2} \sin (\phi-\pi / 2)\right]^{2 / 3} .
$$

If $\phi=\pi / 2$, the P2 bifurcation is still possible. From Eq. (B9) this point is located at

$$
\delta_{1}=1+\delta_{2}^{2}
$$

for small $\delta_{2}$.

\section{SUMMARY AND DISCUSSION}

There is a substantial amount of research in nonlinear systems subjected to secondary sinusoidal modulations. Advantageous effects on the system dynamics, such as control of a chaotic trajectory, can occur as a result of this type of simple perturbations. This is notably appealing for the case of high speed systems, such as semiconductor lasers that operate in the GHz regime, where strict time constraints limit the computations required for theoretical controlling influences.

As a prelude to investigations of this nature, we have studied a neodymium fiber laser that is operating in the vicinity of its $\mathrm{P} 2$ bifurcation point. This is a complex system in that there a number of longitudinal modes operating, two polarization eigenstates, and possibly saturable absorption effects. However, since its relaxation oscillations are only in the $\mathrm{kHz}$ regime, we are able to make detailed time and frequency domain investigations. In this paper, we have employed the diode laser pump in order to modulate the energy applied to the laser. The primary driver is a $2 \pi / \omega$ periodic component whose amplitude $I_{1}$ is used as a bifurcation pa- rameter. We then considered the consequence of an additional small $\pi / \omega$ periodic perturbation. (The amplitude, $I_{2}$, of the $\omega / 2$ component is on the order of $3 \%$ of $I_{1}$.)

Constructed experimental bifurcation diagrams that encapsulate the ongoing changes in the system dynamics reveals the evolution of the laser with respect to the increase in $I_{2}$. The second tone breaks the period two pitchfork bifurcation and unfolds the attractor. Experimentally this is manifested by a splitting of the P1 orbit and a shifting of the P2 bifurcation point. We find that phase difference between the two drivers plays a crucial role. The unfolding is greatest for a phase of 0 and least for a critical phase of approximately $\pi / 2$. Figure 5 shows that this critical phase can negate the effect of the perturbation even for a large value of $I_{2}$. We have also found that when the laser is operating very near its P2 bifurcation point, the gain of the $\omega / 2$ frequency component scales as $-2 / 3$ for a range of values of the amplitude of the second tone. In other regimes, the gain is constant.

Theoretically, we derive an iterative map from the rate equations for a pump modulated class $B$ laser. This map is motivated by the experimental and theoretical observation that slight modulation of the pump or the intracavity losses leads to large amplitude pulsation events, see Fig. 2(a). This map predicts the separation in time of the pulses and the state of the laser at the end of such events. Though our fiber laser cannot be completely described as a class $B$ laser, our experimental studies show that in the vicinity of the P2 bifurcation point, the map's predictions are in good agreement with the experimental observations. Furthermore, because the damping of the laser is quite small, the map can be simplified so that analytical studies of the fixed points can be performed. As a result, we show that the pitchfork period doubling bifurcation is broken and determine specific conditions and scaling laws for this phenomena. The map is also capable of describing the case $\omega_{2}$ nearly equal to $\omega_{1} / 2$, studied in Ref. [13], and which is known to lead to quasiperiodicity. For clarity, we have chosen to focus only on $\omega_{2}=\omega_{1} / 2$.

Our results are in agreement with previous observations on two tone loss modulation of a $\mathrm{CO}_{2}$ laser $[10,11]$ that we now discuss. Glorieux et al. [11] noted the unfolding of the P2 bifurcation transition and examined, experimentally and numerically, the behavior of a gain function $G$, Eq. (3.1). They noted that $G$ follows a $\delta_{2}^{2 / 3}$ scaling law for small values of $\delta_{2}$ but then saturates to a constant as $\delta_{2} \rightarrow 0$. We have also observed this $-2 / 3$ scaling law experimentally. Using our analysis of the imperfect bifurcation problem, we find that $G$ is proportional to $T / \delta_{2}$ where $T$ is defined by Eq. (4.8). For a fixed $\delta_{1} \leqslant 1, T$ behaves as $\delta_{2}$ for $\delta_{2}$ small, but as $\delta_{2}^{1 / 3}$ for larger values of $\delta_{2}$ (specifically when $\left.\delta_{2}=O\left[\left(\delta_{1}-1\right)^{2 / 3}\right]\right)$. Computing $G$, we obtain the scaling laws found in [11]. Note that the relatively large factor multiplying $\delta_{2}$ in Eq. (4.8) explains why $\delta_{2}$ needs to be quite small in order to observe the $\delta_{2}^{1 / 3}$ effects. Corbalan et al. [10] analyzed the shift of the P2 bifurcation transition as a function of $\delta_{2}$ and for a fixed $\delta_{1}$. We identify this shift as the change of the limit point as $\delta_{2}$ increases. This point scales like $\delta_{1}-1=O\left(\delta_{2}^{2 / 3}\right)$. If $\phi \approx \pi / 2$, the scaling is much smaller, and we have found that $\delta_{1}-1=O\left(\delta_{2}^{2}\right)$.

In both [10] and [11], a critical phase is determined for 
which the modification of the unperturbed (that is $\delta_{2}=0$ ) bifurcation diagram is minimum as $\delta_{2}$ is increased. They found this critical phase close to $\pi$. In contrast, we determine this critical phase to be close to $\pi / 2$. The difference results from the fact that the losses are periodically modulated in $[10,11]$ and not the pump as in our study. We have found analytically that the equations for the loss modulated case leads to Eq. (4.8) except that the imperfection term $\delta_{2} \sin (\phi-\pi / 2)$ is replaced by the term $\delta_{2} \cos (\phi-\pi / 2)$ Hence, the imperfection is canceled if $\phi=\pi$. Note that the critical phase found in $[10,11]$ is slightly different from $\pi$ because of the effect of damping which is ignored in our analysis.

In [10], the change of the P4 bifurcation point is also analyzed experimentally. Our experiments on the fiber laser do not allow for accurate observations but analysis of the map is possible. We find that the $\mathrm{P} 4$ bifurcation point is not altered by the second tone as is the P2 bifurcation transition. As a result, the shift of the P4 bifurcation point is simply a linear function of $\delta_{2}$. In conclusion, a minute $\omega / 2$ drive frequency component plays a major role on the fiber laser dynamics at the P2 bifurcation point. This behavior can be accurately predicted by analytical fixed point studies of a map.

\section{ACKNOWLEDGMENTS}

T.C.N. would like to thank the National Research Council for supporting this work. This research was partially supported by the U.S. Air Force Office of Scientific Research Grant No. AFOSR F49620-94-1-0007, the National Science Foundation Grant No. DMS-9308009, the Fonds National de la Recherche Scientific (Belgium), and the InterUniversity Attraction Pole of the Belgian government. The authors would like to thank Pierre Glorieux for illuminating discussions.

\section{APPENDIX A: EQUATIONS FOR THE MAP}

Here we formulate the equations of the map from the pump modulated rate equations of a class $B$ laser. The method is described in detail for a loss modulated class $B$ laser in Ref. [20]. Below we summarize the main steps of the analysis for the pump driven case. We first change variables in the rate equations by introducing the new variables $s, x$, and $y$ defined by

$s \equiv \omega_{R} t, \quad I=|E|^{2}=\left(A_{0}-1\right)(1+y), \quad$ and $\quad N=1+\frac{\omega_{R}}{2} x$.

Equation (4.2) is then rewritten as

$$
\begin{gathered}
x^{\prime}=-y+\delta_{1} \cos \left(\omega_{1} s\right)+\delta_{2} \cos \left(\omega_{2} s+\phi\right) \\
-\epsilon x\left[1+\left(A_{0}-1\right)(1+y)\right], \\
y^{\prime}=(1+y) x,
\end{gathered}
$$

where

$$
\begin{gathered}
\delta_{j} \equiv \frac{A_{j}}{A_{0}-1}, \quad \omega_{j} \equiv \frac{\omega}{j \omega_{R}}(j=1,2), \quad \text { and } \\
\epsilon \equiv\left(\frac{\gamma}{2\left(A_{0}-1\right)}\right)^{1 / 2} .
\end{gathered}
$$

We next determine the solution of these equations by the method of matched asymptotic expansions [24]. Specifically, the solution consists of two main contributions corresponding to the silent and active phases of the intensity oscillations, see Fig. 2(b). We start with the initial condition

$$
x\left(s_{n}\right)=x_{n}<0, \quad y\left(s_{n}\right)=0
$$

and follow a complete orbit in the phase plane $(x, y)$ until we reach the point

$$
x\left(s_{n+1}\right)=x_{n+1}, \quad y\left(s_{n+1}\right)=0 .
$$

The period of the orbit is given by $s_{n+1}-s_{n}$ which satisfies the transcendental equation

$$
\begin{aligned}
s_{n+1}-s_{n}= & {\left[x_{n}-\frac{1}{\varepsilon}-\frac{\delta_{1}}{\varepsilon \omega_{1}} \sin \left(\omega_{1} s_{n}\right)-\frac{\delta_{2}}{\varepsilon \omega_{2}} \sin \left(\omega_{2} s_{n}+\phi\right)\right] } \\
& \times\left\{\exp \left[-\varepsilon\left(s_{n+1}-s_{n}\right)\right]-1\right\} \\
& +\varepsilon\left\{\frac{\delta_{1}}{\omega_{1}^{2}}\left[\cos \left(\omega_{1} s_{n+1}\right)-\cos \left(\omega_{1} s_{n}\right)\right]\right. \\
& \left.-\frac{\delta_{2}}{\omega_{2}^{2}}\left[\cos \left(\omega_{2} s_{n+1}+\phi\right)-\cos \left(\omega_{2} s_{n}+\phi\right)\right]\right\} \\
& +O\left(\varepsilon^{2}\right)
\end{aligned}
$$

while the change of $x$ after one orbit is obtained from

$$
\begin{aligned}
x_{n+1}= & -\left[\frac{1}{\varepsilon}+\left(x_{n}-\frac{1}{\varepsilon}\right) \exp \left[-\varepsilon\left(s_{n+1}-s_{n}\right)\right]\right. \\
& +\frac{\delta_{1}}{\omega_{1}}\left\{\sin \left(\omega_{1} s_{n+1}\right)-\exp \left[-\varepsilon\left(s_{n+1}-s_{n}\right)\right] \sin \left(\omega_{1} s_{n}\right)\right\} \\
& +\frac{\delta_{2}}{\omega_{2}}\left\{\sin \left(\omega_{2} s_{n+1}+\phi\right)-\exp \left[-\varepsilon\left(s_{n+1}-s_{n}\right)\right]\right. \\
& \left.\left.\times \sin \left(\omega_{2} s_{n}+\phi\right)\right\}+O\left(\epsilon^{2}\right)\right]
\end{aligned}
$$

Finally, the maximum value of the intensity variable $y$ is related to $x_{n+1}$ and is given by

$$
\max (y)=\frac{1}{2} x_{n+1}^{2} \text {. }
$$

\section{APPENDIX B: PERIOD-TWO SOLUTION}

The equations for a period-two solution satisfying the two conditions $s_{n+2}-s_{n}=4 \pi \omega_{1}^{-1}$ and $x_{n+2}=x_{n}$ are given by

$$
s_{n+1}-s_{n}=-2 x_{n}+2 \delta_{1} \omega_{1}^{-1} \sin \left(\omega_{1} s_{n}\right)+2 \delta_{2} \omega_{2}{ }^{-1}
$$$$
\times \sin \left(\omega_{2} s_{n}+\phi\right),
$$ 


$$
\begin{aligned}
x_{n+1}-x_{n}= & -\delta_{1} \omega_{1}^{-1}\left[\sin \left(\omega_{1} s_{n+1}\right)+\sin \left(\omega_{1} s_{n}\right)\right] \\
& -\delta_{2} \omega_{2}^{-1}\left[\sin \left(\omega_{2} s_{n+1}+\phi\right)+\sin \left(\omega_{2} s_{n}+\phi\right)\right], \\
s_{n}+4 \pi \omega_{1}^{-1}-s_{n+1}= & -2 x_{n+1}+2 \delta_{1} \omega_{1}^{-1} \sin \left(\omega_{1} s_{n+1}\right) \\
& +2 \delta_{2} \omega_{2}^{-1} \sin \left(\omega_{2} s_{n+1}+\phi\right), \\
x_{n}-x_{n+1}= & -\delta_{1} \omega_{1}^{-1}\left[\sin \left(\omega_{1} s_{n}\right)+\sin \left(\omega_{1} s_{n+1}\right)\right] \\
& -\delta_{2} \omega_{2}^{-1}\left[\sin \left(\omega_{2} s_{n}+\phi\right)+\sin \left(\omega_{2} s_{n+1}+\phi\right)\right] .
\end{aligned}
$$

Comparing Eq. (B2) and Eq. (B4) indicates that

$$
\begin{gathered}
\delta_{1}{\omega_{1}}^{-1}\left[\sin \left(\omega_{1} s_{n+1}\right)+\sin \left(\omega_{1} s_{n}\right)\right]+\delta_{2} \omega_{2}{ }^{-1} \\
\times\left[\sin \left(\omega_{2} s_{n+1}+\phi\right)+\sin \left(\omega_{2} s_{n}+\phi\right)\right]=0
\end{gathered}
$$

and

$$
x_{n+1}=x_{n} .
$$

Adding Eq. (B1) and Eq. (B3) then gives

$$
x_{n}=-\pi \omega_{1}^{-1} \text {. }
$$

Then using Eq. (B7), we rewrite Eq. (B1) and Eq. (B3) as

$$
\begin{aligned}
s_{n+1}-s_{n}= & 2 \pi \omega_{1}^{-1}+2 \delta_{1} \omega_{1}^{-1} \sin \left(\omega_{1} s_{n}\right) \\
& +2 \delta_{2} \omega_{2}^{-1} \sin \left(\omega_{2} s_{n}+\phi\right), \\
s_{n}-s_{n+1}= & -2 \pi \omega_{1}^{-1}+2 \delta_{1} \omega_{1}^{-1} \sin \left(\omega_{1} s_{n+1}\right) \\
+ & 2 \delta_{2} \omega_{2}^{-1} \sin \left(\omega_{2} s_{n+1}+\phi\right) .
\end{aligned}
$$

These equations are two equations for the unknowns $s_{n+1}$ and $s_{n}$. With $S=\omega_{1} s_{n}$ and $T=\omega_{1}\left(s_{n+1}-s_{n}\right)-2 \pi$, Eq. (B8) becomes

$$
\begin{aligned}
T= & 2 \delta_{1} \sin (S)+4 \delta_{2} \sin \left(\frac{1}{2} S+\phi\right), \\
& -T=2 \delta_{1} \sin (S+T)-4 \delta_{2} \sin \left[\frac{1}{2}(S+T)+\phi\right] .
\end{aligned}
$$

Equation (B9) captures the essential dynamics of the P2 bifurcation point.

We are interested in solving Eqs. (B9) for small $\delta_{2}$ and for $\delta_{1}$ near the $\mathrm{P} 2$ bifurcation point $\delta_{1}=1$. Therefore, treat $\delta_{2}$ as an imperfection in the bifurcation problem and seek a solution of the form [25]

$$
\begin{gathered}
T=\delta_{2}^{1 / 3} T_{0}+\delta_{2} T_{3}+\cdots, \\
S=-\pi+\delta_{2}^{1 / 3} S_{0}+\delta_{2}^{2 / 3} S_{3}+\cdots
\end{gathered}
$$

We also need to expand the bifurcation parameter as

$$
\delta_{1}=1+\delta_{2}^{2 / 3} \Delta+\cdots
$$

where $\Delta$ is the new control parameter. Substituting these expressions into Eq. (B9) leads to a succession of equations for the unknown coefficients in Eq. (B10) and Eq. (B11). Analyzing these problems and their solvability conditions leads to the following results:

$$
S_{0}=-\frac{1}{2} T_{0}
$$

where $T_{0}$ satisfies the following:

$$
2 \Delta T_{0}-\frac{1}{12} T_{0}^{3}+8 \sin (\phi-\pi / 2)=0 .
$$

A plot of $T_{0}$ versus $\Delta$ is shown in Fig. 8 for $\phi=89.91^{\circ}$. This plot shows that the pitchfork bifurcation is broken. The lower branch describes the evolution of the P1 orbit while the upper branch descends towards the limit point. The solution of Eq. (B14) is analyzed in Sec. III. If $\phi=\pi / 2$, the imperfection term in Eq. (B14) disappears and the two tone modulation problem reduces to the perfect bifurcation case. We may analyze this problem using Eq. (B9) with $\phi=\pi / 2$. The $\mathrm{P} 2$ bifurcation point, $\delta_{1}=\delta_{p d}$ is then obtained from the linearized problem for $(T, S)=(0,-\pi)$. We find that it is the root of

$$
\delta_{p d}^{2}-\delta_{p d}-\delta_{2}^{2}=0
$$

Hence, when $\delta_{2}$ is small, we have

$$
\delta_{p d} \cong 1+\delta_{2}^{2} \text {. }
$$

[1] F. T. Arecchi, G. L. Lippi, G. P. Puccioni, and J. R. Tredicce, Opt. Commun. 51, 308 (1984).

[2] F. T. Arecchi, R. Meucci, G. Puccioni, and J. Tredicce, Phys. Rev. Lett. 49, 1217 (1984).

[3] V. N. Chizhevsky and S. I. Turovets, Phys. Rev. A 50, 1840 (1994).

[4] V. N. Chizhevsky and P. Glorieux, Phys. Rev. E 51, R2701 (1995).

[5] Z. Gills, C. Iwata, R. Roy, I. B. Schwartz, and I. Triandaf, Phys. Rev. Lett. 69, 3169 (1992).

[6] P. Colet and R. Roy, Opt. Lett. 19, 2056 (1994).

[7] P. M. Alsing, A. Gavrielides, V. Kovanis, R. Roy, and K. S. Thornburg, Jr., Phys. Rev. E (to be published).
[8] K. Wiesenfeld and B. McNamara, Phys. Rev. Lett. 55, 13 (1985).

[9] B. Derighetti, M. Ravani, R. Stoop, P. F. Meier, E. Brun, and R. Badii, Phys. Rev. Lett. 55, 1746 (1985).

[10] R. Corbalan, J. Cortit, A. N. Pisarchik, V. N. Chizhevsky, and R. Vilaseca, Phys. Rev. A 51, 663 (1995).

[11] P. Glorieux, C. Lepers, R. Corbalan, J. Cortit, and A. N. Pisarchik, Opt. Commun. 118, 309 (1995).

[12] V. N. Chizhevsky and R. Corbalan, Phys. Rev. A 53, 1830 (1996).

[13] Y. Braiman and I. Goldhirsch, Phys. Rev. Lett. 66, 2545 (1991).

[14] P. Colet and Y. Braiman, Phys. Rev. E 53, 200 (1996). 
[15] R. Meucci, W. Gadomski, M. Ciofini, and F. T. Arrecchi, Phys. Rev. E 49, R2528 (1994).

[16] M. Ciofini, R. Meucci, and F. T. Arecchi, Phys. Rev. E 52, 94 (1995).

[17] S. T. Vohra, L. Fabiny, and F. Bucholtz, Phys. Rev. Lett. 75, 65 (1995).

[18] The fiber laser undergoes a supercritical pitchfork bifurcation in which the $P 1$ fixed point becomes unstable and two stable symmetric fixed points appear. See S. H. Strogatz, Nonlinear Dynamics and Chaos (Addison-Wesley, Reading, MA, 1994), p. 55.

[19] B. Derighetti, M. Ravani, R. Stoop, P. F. Meier, E. Brun, and R. Badii, Phys. Rev. Lett. 55, 1746 (1985).
[20] I. B. Schwartz and T. Erneux, SIAM (Soc. Ind. Appl. Math.) J. Appl. Math. 54, 1083 (1994).

[21] D. Derozier, S. Bielawski, and P. Glorieux, Opt. Commun. 83, 97 (1991)

[22] S. Bielawski, D. Derozier, and P. Glorieux, Phys. Rev. A 46, 2811 (1992).

[23] D. Dangoisse, J. C. Celet, and P. Glorieux, Phys. Rev. E 56, 1396 (1997).

[24] J. Kevorkian and J. D. Cole, Perturbation Methods in Applied Mathematics, Applied Mathematical Sciences Vol. 34 (Springer-Verlag, Berlin, 1984).

[25] B. J. Matkowsky and E. L. Reiss, SIAM (Soc. Ind. Appl. Math.) J. Appl. Math. 33, 230 (1977). 